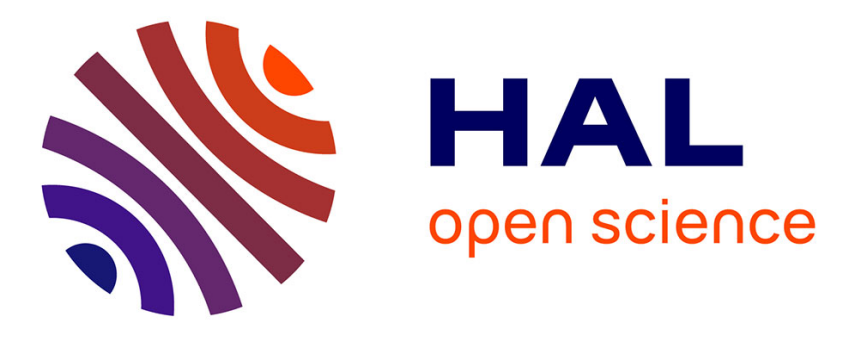

\title{
La fabrique de la langue, fabrique de l'humain
}

Didier Bottineau

\section{To cite this version:}

Didier Bottineau. La fabrique de la langue, fabrique de l'humain. K. Nassikas, E. Prak-Derrington, C. Rossi. Fabriques de la langue, Presses Universitaires de France, pp.161-197, 2012, 978-2-13-059126-9. hal-00770370

\section{HAL Id: hal-00770370 https://hal.science/hal-00770370}

Submitted on 5 Jan 2013

HAL is a multi-disciplinary open access archive for the deposit and dissemination of scientific research documents, whether they are published or not. The documents may come from teaching and research institutions in France or abroad, or from public or private research centers.
L'archive ouverte pluridisciplinaire HAL, est destinée au dépôt et à la diffusion de documents scientifiques de niveau recherche, publiés ou non, émanant des établissements d'enseignement et de recherche français ou étrangers, des laboratoires publics ou privés. 
Didier Bottineau

CNRS, MoDyCo, Université Paris Ouest - Nanterre - La Défense

\section{La fabrique de la langue, fabrique de l'humain}

La rencontre qui a suscité la présente étude posait dans sa présentation la question suivante : «Où et comment se fabrique la langue ? Quels sont les lieux où elle se crée, se re-crée et se modifie constamment ?». Ainsi formulée, cette question présente l'intérêt d'articuler de manière atypique et de déconstruire implicitement trois évidences présupposées, « la langue », « se fabrique » et « où »/ en des « lieux » :

(i) « la langue » subsume ici ce que l'on distingue traditionnellement par les termes langage, langue et parole :

- le langage en tant que faculté propre à l'espèce, système éthologique humain opposable aux pratiques de communication animale ;

- les langues en tant que disciplines communautaires et systèmes cohérents, opposables entre eux (le français, l'anglais...) au sein de l'espèce humaine, de routines langagières orchestrables pour la production du sens et la cohésion des groupes ;

- la parole en tant que pratique interactionnelle, manifestation empirique d'un enchaînement verbal prenant pour ressource le système de possibilités et de contraintes présenté par une langue donnée.

(ii) «se fabrique » subsume ici ce que l'on distingue traditionnellement par les termes d'origine et de diachronie :

- origine du langage : il existerait un seuil évolutionnaire au-delà duquel on observerait, dans le devenir de l'espèce humaine, un sous-système éthologique cohérent et fermé, caractérisé par des classes de comportements (cris articulés, mots-phrases) à effets sémiotiques ; selon les théories, l'apparition de ce dispositif serait lié à l'évolution du cerveau (son volume et sa structure), à l'évolution du corps (redressement, station debout, marche, descente du larynx), à l'évolution des rapports à l'environnement liées ou non à celles du cerveau et du corps (mobilité et exploration de l'espace, libération des membres antérieurs et préhension des objets, modification de la perspective du champ visuel et de l'adressage des vocalisations, facilitation de l'interactionnalisation des rapports sociaux), à l'exaptation d'organes détournés de leurs fonctions initiales (respiration, morsure, mastication, succion) et coordonnés au profit de fonctions innovantes (l'articulation phonatoire) en relation avec des gestualités corrélées (la préhension), etc. Et ce sous-système éthologique fournirait un avantage adaptatif lié à diverses fonctions telles que l'exercice des rapports de force dans l'intersubjectivité et la coordination de communautés pour la conquête de territoires et de ressources alimentaires et reproductives.

- origine des langues: les langues proliféreraient comme par division et spécialisation cellulaire (latin / langues romanes; Indo-Européen reconstruit / satem et centum), éventuellement depuis une langue unique (monogénétique) selon certains auteurs (dont Ruhlen), et certaine disparaîtraient comme par mort cellulaire (le gaulois, si tant est qu'il ait réellement disparu ${ }^{1}$ ). Les langues formeraient des objets aux contours définis et évolutifs,

\footnotetext{
${ }^{1}$ La thèse de référence est que le gaulois aurait disparu vers le $2^{\mathrm{e}}$ siècle et que le breton serait issu du cornique britannique ultérieurement, réintroduisant en Armorique une langue celtique après la disparition du gaulois. Mais
} 
comparables aux êtres vivants, au point que la question de leur origine puisse être posée. La corrélation de l'origine du langage et des langues se décline elle-même en une multitude d'approches ${ }^{2}$.

- diachronie : toute langue est munie d'une histoire et «évolue » au niveau de chacune de ses composantes (système phonologique, accentuel et prosodique; lexique, morphologie, syntaxe) sous l'effet de moteurs systémiques (auto-organisationnels) et/ou situés (dans la pragmatique des contacts intersubjectifs et intercommunautaires : faits de langues en contacts, emprunts, calques, hybridations, créolisations ; dans la déformation progressive des pratiques par les générations successives de locuteurs comme dans les ruptures et effets de seuils motivés par des évènements historiques critiques). La diachronie comprend la microdiachronie : chaque interaction verbale, locale dans l'espace et dans le temps, est l'occasion possible des hapax innovants réussis qui finissent par s'inscrire dans le devenir du système.

(iii) « où » et «lieux » subsume les niveaux d'observation et de modélisation en lesquels on peut s'intéresser à la «langue » dans les acceptions précédemment évoquées : le sujet dans son rôle de producteur / interprétant dans le cadre de l'interaction; le sujet dans son rôle d'apprenant et de contributeur plus ou moins créatif dans le cadre de l'acquisition et du développement; les groupes et communautés sociolinguistiques à géométrie variable dans l'espace et dans le temps dans le cadre de la pragmatique des micro- et macro-interactions; le système en tant qu'il est décrit par des spécialistes et prescrit par une autorité institutionnelles; et le mythe de la langue en tant qu'objet hybride amalgamant différents niveaux de manifestation dans le cadre de discours profanes et experts, porteurs de motivations identitaires et politiques.

Si la question où focalise l'attention sur les manifestations de l'interaction (la parole), le verbe fabrique focalise l'attention sur l'origine du langage ou des langues, mais s'applique à la langue en tant que référent commun présent pour les interlocuteurs. Le terme la langue est ici inscrit dans une construction inhabituelle et hybride, dont les différentes parties invoquent tour à tour ce qui correspond à la parole, aux langues et au langage. Cette situation correspond à peu près à ce qu'en linguistique cognitive on nomme intégration conceptuelle (conceptual blending : Fauconnier \& Turner 1998), et elle conduit à une renégociation du sens du mot en fonction du contexte avec lequel il interagit. Ce principe remet en cause l'idée de niveaux $\mathrm{d}$ 'analyse et des objets discrets : le complexe langage / langue (s) / parole fonctionne comme une «fabrique », un processus dynamique en réseau distribué sur le sujet expérient et pensant, les sujets interagissants, les groupes, les communautés, et l'environnement matériel et symbolique aux différentes échelles temporelles correspondantes. Comprendre la « fabrique de la langue », c'est produire une vision d'ensemble de ce processus et modéliser les effets structurants qu'il implique dans l'expérience et le devenir des sujets, des groupes et de l'espèce. Dans les lignes qui suivent, nous tentons de donner un aperçu de cette articulation à partir de l'exemple fourni par la question initiale, le mot « langue » lui-même.

\section{La fabrique du mot}

\footnotetext{
que le gaulois ait réellement disparu à ce moment est incertain, et le breton actuel pourrait résulter d'une rencontre des deux langues.

2 Pour Ruhlen 1994, la langue-mère correspondrait à une langue qui à un moment donné s'est imposée à l'exclusion des autres, au point que toutes les langues actuelles en dérivent. Ce modèle ne conjoint pas la languemère à l'origine des langues ni l'origine du langage. En Azerbaïdjan, Mammadov propose que l'unité de base de la production phonatoire, le phonème, vale successivement pour la phrase, le syntagme, le mot, puis le morphème dans l'histoire évolutive d'une langue donnée : ce modèle indexe le processus d'émergence d'une langue sur celui de l'évolution de la sémiotisation du cri articulé.
} 
On envisage traditionnellement un mot comme un «objet » isolable et caractérisable par sa composition propre. Dans le modèle saussurien, il s'agit de deux représentations mentales : le signifiant («l'image acoustique» et le signifié («concept»). Ce couple peut être mis en rapport avec une troisième entité mentale, le «référent »: la représentation d'une occurrence réelle ou imaginaire, perçue en situation ou capturée dans le discours, de portée spécifique ou générique. En utilisant le mot langue, on croit produire une occurrence de cette triade. Or, dans l'expérience humaine de la parole, le mot se présente rarement comme un évènement isolé. Cela peut se produire avec l'interpellation (Eponine!), l'insulte (idiot!), certaines injonctions (Entre! Dehors!), certaines réactions émotionnelles (Bof! Lol! Ridicule! Délicieux! Miam!), certaines étapes d'interactions routinières comme le jeu (Carte! Pénalty! Echec!). En dehors de ces contextes, le mot est un fragment de la parole de quelqu'un, un extrait du discours de soi ou d'autrui, un segment d'action phonatoire et d'expérience perceptuelle et interprétative. Considérer le mot en tant qu'unité hors de ce jeu dialogique multicontextuel, c'est extraire un échantillon de son environnement dynamique à des fins d'analyse et de mesure et en modifier les propriétés constitutives par cet acte même.

- Du côté du signifiant, les langues marquent diversement cette relativité lexique / contextes phrastiques: une langue à cas comme le latin marque par une flexion casuelle finale l'insertion du mot dans le réseau syntaxique (amicus, amicum...), et il n'existe pas de forme stabilisée antérieure à l'insertion (*amic-), pas même pour l'interpellation (vocatif amice). Une langue comme le breton modifie la consonne initiale du mot en fonction de l'introducteur grammatical qui le précède : mamm «mère », ma vamm «ma mère »; tad «père », ma zad «mon père ». Une langue agglutinante comme l'inuktitut produit en discours des unités lexicales en combinant une racine non libre à une chaîne de suffixes grammaticaux qui en profilent le sens de manière détaillée. Dans tous ces cas, le signifiant lexical est formellement porteur de sa relativité discursive : l'idée même qu'il existe un «mot»comme catégorie commune à $\mathrm{tad}$ et zad n'est pas une évidence première lorsque l'on considère la réalité pratique de l'émergence et l'emploi du «mot», lequel apparaît comme une construction de linguiste. Il ne s'agit pas de nier que cette unité existe, car une démarche distributionnelle raisonnée permet en effet de la reconstruire, en l'occurrence en formalisant les règles de mutation de la consonne initiale, qui sont organisées de manière cohérente et constituent un savoir-faire épilinguistique chez les locuteurs (non explicité, non connu réflexivement, mais systématiquement mis en œuvre: Culioli 1968). Mais il s'agit d'intégrer le fait que les occurrences empiriques des formes du mot lexical sont variables, que cette variation peut être grammaticalisée et formalisée à l'écrit, ou ne pas l'être mais se manifester à l'oral (j'vais'm'couper les'ch'feux). Cette variation est emblématique du fait qu'un mot est avant tout une composante ajustable d'un comportement individuel coordonné et contextualisé par une source subjective, une relation intersubjective et une inscription situationnelle, à la fois matérielle et symbolique, immédiate et large. Demandons-nous donc à présent ce que devient le signifié en sémantique lexicale dès lors que cette variation est prise en compte.

- Du côté du signifié, définir le mot comme extrait de discours de soi ou d'autrui permet de distinguer l'occurrence (l'exemple) de la classe (l'exemplaire). Le mot-exemple, contextualisé dans un énoncé formulé par un parleur dans une situation interactionnelle et matérielle, par exemple Ma fille a pris anglais première langue, prend son sens local et momentané dans le réseau des relations intra- et extraverbales où il s'inscrit en tant que comportement énonciatif (la production phonatoire) et interprétatif (la perception auditive et autre, y compris par le locuteur lui-même, réflexivement). L'inventaire des sens locaux constitutifs de la polysémie générale du mot-exemplaire s'obtient en collectant l'ensemble des interactions verbales susceptibles de faire émerger une interprétation ciblée du mot-exemple : J'adore la langue de bruf, Le visage-pâle a la langue fourchue, Ne sois pas mauvaise langue / Langue de vipère!, 
Les chats ont la langue râpeuse, Nous avons pris langue, Un tombolo est une langue de sable reliant une île au continent / Le sillon de Talbert est une langue de galets à la jonction des estuaires $d u$ Trieux et $d u$ Jaudy. Chacun de ces exemples mobilise une acception contextuelle: l'organe vivant chez l'homme ou l'animal, l'aliment, l'organe actif comme symbole de la parole dans son aptitude à révéler l'état d'esprit d'un individu, l'organe comme dénomination métonymique de l'ensemble de pratiques constitutif d'un système de parole comme le «français », la langue comme «matière » ou discipline scolaire, la langue comme avancée géographique. A cela s'ajoutent des acceptions expertes récentes, telles que l'opposition langue / parole chez Saussure ou langue / discours chez Guillaume : La langue est un fait social (? Le français est un fait social); La langue est le niveau de la représentation et le discours celui de l'expression (psychomécanique du langage). Chaque énoncé illustratif focalise l'attention interprétante sur l'une des valeurs d'exemple à l'exclusion des autres, qui peut-être sont secondairement activées dans une sorte d'arrièreplan mental mémoriel, mais cela ne va pas de soi : rares sont les sujets interrogés qui admettent avoir jamais envisagé consciemment l'existence d'un rapport entre la langue comme organe et la langue comme discipline scolaire ; et la reconnaissance de ce rapport n'est nullement nécessaire pour le bon fonctionnement des mots-exemples dans les énoncés respectifs.

Le mot langue n'apparaît presque jamais isolément : il n'existe pas de situation d'interaction verbale spontanée où l'on s'exclame Langue! en guise d'interjection, de réponse à un stimulus émotionnel, d'injure, d'injonction, d'exécution de routine ludique, etc. Tout au plus entendra-t-on Langue(s) (?) en guise de question elliptique lors d'entrevues ou dans des documents administratifs où l'on décline ses compétences. En revanche, il est possible d'amener l'attention interprétative à réaliser une synthèse des valeurs d'exemples mémorisées dans leur diversité en faisant du signifiant langue un emploi dans un contexte thématisant qui, justement, soulève la question du rapport exemple / exemplaire, exactement comme le fait le titre du colloque: Fabriques de la langue pluralise de manière inattendue le mot fabrique, d'usage assez récent en sciences du langage (cf. le colloque La fabrique du signe, Toulouse, IRPALL, 12-13 octobre 2006, et la même année le numéro 28/2 de la revue Histoire Epistémologie Langage intitulé Hyperlangues et fabriques de la langue par Auroux et Mazière); et la langue singularise de manière inattendue un terme que l'on attendait dans sa diversité quantitative (la fabrique des langues) et/ou qualitative (la fabrique du langage et des langues par la parole). En ce qui me concerne, la première lecture du titre du colloque a suscité une interrogation sur le sens de fabriques et de la langue en réaction à la construction atypique qui les conjoint : ce titre a agi de manière performative sur le mot langue lui-même, activant la problématique qu'il thématise. Ce processus s'observe régulièrement en acquisition de la langue maternelle ${ }^{3}$.

Lorsque qu'un linguiste modélise l'invariant sémantique du mot lexical, il regroupe les valeurs-exemples et produit une synthèse de traits hétérogènes mais cohérents, que Culioli 1981 / 1990 nomme la notion, en insistant sur la dimension matérielle et abstraite, symbolique et culturelle des éléments impliqués. La sémantique cognitive y voit une catégorie munie d'un centre auquel diverses valeurs sont adjoignables par divers processus cognitifs individuels et

\footnotetext{
${ }^{3}$ Comme dans l'anecdote suivante : un grand-père, retardé par des embouteillages, manifeste son exaspération. Sa petite fille de deux ans s'exclame: Sélebodel! Elle a simplement reproduit ce qui pour elle est une interjection motivée par l'exaspération, qu'elle a reconnue et partagée par empathie. Le grand-père reconnaît le modèle adulte C'est le bordel! , manifeste son étonnement, répète le mot incriminé en le recontextualisant, le constitue en objet, le recharge sémantiquement en le manipulant et en manifestant un intérêt inattendu pour l'enfant, qui reçoit par la même une récompense émotionnelle de nature à susciter l'envie de retester la chose. L'acquisition de la langue passe par de tels enchaînements vertueux de malentendus et de renégociations involontaires où se jouent l'émotion et l'affect.
} 
empruntés à la psychologie (la métaphore, le transfert inter-domaines, l'intégration conceptuelle, le fenêtrage attentionnel).

Si l'on prend en compte la profondeur dialogique des sources énonciatives des valeurs extraites des discours, on précise que cette diversité des effets de sens s'explore à travers l'expérience des interactions verbales hétérogènes qui la vectorisent, lesquelles rendent nonnécessaire l'hypothèse d'un invariant fédérateur initial et la dérivation des valeurs réputées périphériques à partir d'une primitive. Cette synthèse est provoquée a posteriori par la thématisation du mot dans un contexte saillant, voire anormal ; à la limite, la notion naît par appropriation réflexive à l'instant même où l'on s'interroge sur son existence ${ }^{4}$.

On aboutit donc à une sorte d'aporie : la cohérence notionnelle du sens lexical suggère que sa formation a fait l'objet d'une démarche psychologique cohérente par le sujet qui fait l'expérience individuelle du monde physique par sa motricité et sa perception multimodale, mais l'expérience des interactions verbales tend à supplanter le rapport individuel sujet / monde et livre les valeurs concernées à travers un tissu dialogique qui rend inutile, voire impossible, le modèle d'expérience personnelle par lequel ces représentations seraient susceptibles de se former. En cela, la parole, l'ensemble des interactions verbales, incrustent dans l'environnement sensible des processus qui sont «interprétables », sémiotisables comme des modèles d'organisation psycho-sémantique du lexique qui pourraient correspondre à ce à quoi on aboutirait si on vivait personnellement l'ensemble des expériences relayées par les discours correspondants. La parole, en tant qu'intervension corporelle perturbatrice dans le monde dont les corps eux-même, est un vecteur distribué de la cognition et, étayée par sa profondeur dialogique, elle simule des architectures sémantiques acceptées par les sujets parce que ressenties comme cohérentes et conformes aux fonctionnements subjectifs : ces contrefaçons réussies sont indétectables.

Par exemple, la cohérence affichée par le mot langue ne correspond pas à une représentation produite dans sa globalité par chacun des sujets usagers du terme. Cette cohérence se manifeste de manière dispersée au gré des interactions verbales où chaque participant peut faire évoluer le réseau en apportant sa petite contribution personnelle, souvent conformiste, parfois originale, comme dans cette plaisanterie personnelle: l'anglais est une langue perfide ; le français, une langue fourchue, l'arabe, une langue râpeuse. "râpeuse » se dit normalement de la langue du chat ou de la girafe et évoque une sensation tactile, sans réinvestissement symbolique particulier autre que le déplaisir de la friction ; «fourchu » se dit de la langue sifflante du serpent, avec comme connotation possible la duplicité malveillante cf. Kââ dans Le Livre de la Jungle - et la projection anthropomorphique ; «perfide » se dit d'Albion et d'une mentalité collective que l'on croit retrouver dans la parole des individus concernés; et la boutade réalise une intégration conceptuelle en faisant converger les propriétés de trois acceptions hétérogènes sur un support unifiant, les «langues » en tant qu'ensembles de pratiques formelles régulées, avec les effets synesthésiques que cela induit.

Le linguiste (l'écrivain, le poète, l'humoriste, le philosophe, le psychanalyste, l'avocat, le politique...) peut ainsi par sa démarche susciter une prise de conscience synthétique de la cohérence sémantique des usages collectifs et en inférer (à tort ?) qu'elle est emblématique de la cohérence de processus mentaux individuels. Pour que les interactions verbales constatées soient effectivement produisibles et intelligibles, il suffit que chaque individu réalise des

\footnotetext{
${ }^{4}$ Le rapport phénoménologique à l'écrit, par la lecture comme par l'écriture et leur apprentissage, joue un rôle moteur dans ce processus en confrontant l'expérient à une manifestation discontinuiste de la parole - les mots écrits sont séparés et posés comme unités robustes et stabilisées, en contraste avec le continuum flexible de l'oralité fugace; le sens du mot écrit connaît le même effet de figement, avec les conséquences que cela implique pour l'accès à la connaissance (Goody 1979).
} 
contributions conformes ou déviantes selon des modalités elles-mêmes régulées et identifiables. Chaque occurrence renouvelée du mot langue est à la fois analogue aux précédentes et/ou décalée selon un processus subjectif socialement régulé mis en œuvre dans l'interaction. La somme de ces rapports fournit une «représentation », un modèle cumulatif correspondant à la connaissance de la sémantique lexicale que l'on acquiert en participant à toutes les interactions, ce que tente de faire le linguiste en se munissant de corpus ${ }^{5}$. Les sujets, eux, forment des notions personnelles en participant dans leur expérience relative, sous leur angle d'attaque social psychosocial, leur propre version de la notion, et par leur engagement verbal, ils contribuent personnellement à la faire évoluer en recourant aux mêmes processus de «fabrication » que les autres participants dans la communauté. In fine, la somme totale des contributions, regroupées par le linguiste, fournit une simulation non réaliste, mais plausible, d'une dynamique cognitive pseudo-individuelle que nul n'a personnellement centralisée. Chaque locuteur s'engage comme joueur de la partie collective de parole dans un rôle déterminé, et aucun ne totalise à lui seul une représentation complète de l'esprit de l'équipe, exactement comme dans les vols d'étourneaux aucun oiseau n'intériorise un modèle cognitif de sa position dans la dynamique du groupe.

On considère donc le mot comme une «madeleine sociale»: un acte de réminiscence suscitée par une action intentionnelle, une clé vocale permettant l'activation d'un processus cognitif de nature biosémiotique (Hoffmeyer 2008). On formule la chose de manière binariste en séparant le «corps» de «l'esprit» parce que la phraséologie disponible oriente continuellement le propos dans ce sens (Keller 2006, Bottineau 2010b), mais on considère que les processus biomécaniquement incarnés et cognitifs ne font qu'un, et que le signal, la sensorimotricité et la neuromotricité sont des manifestations distribuées (sur «le monde», « le corps » et « le cerveau ») d'une dynamique d'ensemble, « la cognition », elle-même non localisable (Cowley 2009). Par «madeleine», on veut dire, par analogie avec la madeleine proustienne, une expérience perceptuelle capable de provoquer un acte de réminiscence. En l'occurrence, la perception est obtenue par un acte moteur phonatoire : la madeleine est fabriquée en temps réel pour les besoins de la réminiscence intentionnelle à susciter, la récupération d'éléments de sens extraits des discours-source munis d'exemplaires homologues (Lavie 2008), par sondage dialogique. En tant que geste phonatoire, elle est conforme à un modèle de pratique collective et normée: elle est «sociale». L'effet « magdalénien » résulte de la répétition : chaque occurrence du mot rappelle les conditions discursives et interactionnelles dont ses occurrences antérieures ont déjà été un fragment (exactement comme la madeleine rappelle l'expérience située de sa consommation précédente). Le contenu de la réminiscence est non nécessaire en matière de référence à des objets $^{6}$ et son unité se construit à travers l'extraction dialogique d'effets de sens dans les discours où le signifiant se présente. Lors du réemploi du mot, l'unité lexicale convoque la partie du réseau sémantique qui est réactivée par le contexte discursif : appartement à louer (< latin locare) vs Loué soit le Seigneur (< laudare); la polysémie potentielle du mot de « langue » décontextualisé (l'entrée du dictionnaire), ou même l'homonymie liant plusieurs

\footnotetext{
${ }^{5}$ Il est extrêmement difficile de constituer et d'annoter des corpus conçus de manière à fournir des réponses aux questions ici soulevées. Il faut pour cela de l'interaction verbale spontanée, enregistrée et filmée (avec les mimiques, la cogestualité, les contacts visuels), idéalement selon plusieurs personnes phénoménologiques (la $3^{\mathrm{e}}$ comme observateur extérieur, mais aussi la $1^{\mathrm{e}}$ et la $2^{\mathrm{e}}$ comme participants dialogaux : que voit le locuteur quand il parle, quels évènements l'allocutaire lui renvoie-t-il, etc. Sans ces éléments, un corpus écrit ou oral transcrit capture des indicateurs partiels et transduits de certains faits systémiques et comportementaux, mais il ne permet pas de constituer l'expérience qui fonde la cohérence du processus interactionnel.

${ }^{6}$ Pour Saussure, l'arbitraire du signe, ou du rapport signifiant / signifié, signifie le caractère non déterministe de la relation sens / forme, comme l'indique la variation interlinguistique des désignations lexicales du concept d'arbre : anglais tree, allemand Baum, russe derevo.
} 
entrées, est normalement résolue en discours, sauf accident (On ne met jamais trop d'eau dans un réacteur nucléaire! - constat ou prescription, selon la prosodie de l'exclamation) ou fabrication ludique (Dieu soit loué, et s'il est à vendre, achète, c'est une affaire qui monte: loué est pris au sens de «louange», activé par Dieu, mais ensuite achète force la réinterprétation du souvenir de loué comme «louage ») ${ }^{7}$.

L'interprétation de ce genre d'énoncé se solde généralement soit par un échec dû à l'incompréhension, soit par un éclat de rire. Or le rire est un spasme périodique qui mobilise fortement la motricité musculaire, consomme beaucoup d'énergie, et mobilise la proprioception au point de supplanter toute possibilité de production langagière exophasique (par la parole) ou endophasique (par la pensée) : le premier effet du rire est de bloquer la parole sous toutes ses formes. Il survient dans des situations critiques (au sens nucléaire du terme) où l'interprétation est mise à mal par la soudaine prolifération en chaîne de directions sémiotiques conflictuelles incontrôlables : ici louer 1 vs louer 2, mais aussi la maladresse apparente d'un locuteur qui feint de confondre deux acceptions, révélant en réalité son adresse métalinguistique, sa duplicité, et éventuellement son désir d'introduire un élément perturbateur dans une interaction verbale régulée ; l'interprétant ne peut poursuivre toutes les implications à la fois, surtout si leurs étapes intermédiaires commencent à influencer réciproquement leurs conduites respectives. Le rire contagieux résout la crise en interrompant tout calcul et en suspendant l'interaction, et il permet sa réinitialisation sur des bases sémantiquement et émotionnellement assainies. Son utilisation suggère que la parole est un des moteurs corporels de la cognition parmi bien d'autres, qu'un dérapage de la direction verbale de l'idéation est possible, et qu'il existe des vecteurs corporels non verbaux de la cognition capable d'interventions de secours.

L'interprétation du lexique varie en contexte selon les régions du réseau notionnel qui sont activées, et on peut forcer intentionnellement cette variation en temps réel par le jeu de modulations contextuelles comme dans l'exemple qui précède. Soit les questions suivantes, bien connues: De quelle couleur est la neige? - Blanche. Et qu'est-ce que ça boit, une vache? ... la réponse que l'on obtient le plus souvent, même de la part de linguistes cognitivistes mondialement connus (j'ai testé), est: Du lait. - Réfléchissez mieux. Ce phénomène s'explique par l'amorçage (priming) et le dialogisme : (i) on est habitué depuis l'enfance à rencontrer des co-occurrences lexicales vache / boire / lait dans des interactions relatives à la consommation humaine du lait de vache, aussi ce paquet de mot est-il un candidat mémoriel et culturel robuste au réassemblage; (ii) le lait est appelé en réponse (response) par le stimulus blanche plus fortement que la réponse (answer) eau ne l'est par la question initiale. Ce mécanisme est d'autant plus vigoureux que le stimulus d'amorçage émane de la première réponse de celui que l'on cherche à piéger et non de l'interrogateur; en outre, une attitude de méfiance tend à intensifier le recours préventif à la mémoire et aux connaissances valides qu'elle est censée conserver en dépôt - or c'est cette mémoire même qui recueille le trio boire / lait / vache responsable de l'erreur et activé par l'amorçage. Cette plaisanterie a valeur d'expérience reproductible in vivo en milieu interactionnel et

\footnotetext{
${ }^{7}$ L'interprétation de ce genre d'énoncé se solde généralement soit par un échec dû à l'incompréhension, soit à une crise de fou rire. Or le rire est un spasme périodique qui mobilise fortement la motricité musculaire, cconsomme beaucoup d'énergie, et mobilise la proprioception au point de supplanter toute possibilité de production langagière exophasique (par la parole) ou endophasique (par la pensée) : le premier effet du rire est de bloquer la parole. Il survient dans des situations critiques où l'interprétation est mise à mal par des directions sémiotiques conflictuelles : ici louer 1 vs louer 2, mais aussi la maladresse apparente d'un locuteur qui feint de confondre deux acceptions, révélant en réalité son adresse métalinguistique, sa duplicité, et éventuellement son désir d'introduire un élément perturbateur dans une interaction verbale régulée. Le rire contagieux résout la crise en suspendant l'interaction et permet sa réinitialisation sur des bases sémantiquement et émotionnellement assainies. Son utilisation suggère que la parole est un des moteurs corporels de la cognition parmi bien d'autres.
} 
psychosocial : elle fait apparaître la force de la procédure verbale sur le calcul ou la logique et sa capacité à faire tenir des propos organisés par sa cohérence propre au détriment de toute analyse sensée, en laissant même le sentiment que la procédure est ou fait la logique du raisonnement ou du calcul. L'expérience de cette erreur peut s'avérer troublante pour le cobaye, surtout s'il s'agit d'un expert de la cognition computationnelle.

Le mot n'est ni un désignateur d'objet, ni le label d'un concept, d'une catégorie ou d'une représentation robuste. Le mot opère comme un acte : en tant qu'intervension motrice réelle ou simulée (endophasie) sur le monde dont les corps percevants, il permet de coordonner intersubjectivement la focalisation attentionnelle sur une notion dialogique évolutive. Revenons sur l'exemple de la binarité cerveau / esprit, brain / mind en anglais. Dans les deux langues, la même distinction est objectivement évidente parce que deux paires lexicales la constituent en parallèle : à ces deux paires de mots correspond nécessairement une paire d'objets, ou du moins de catégories ou de concepts indépendants du langage et des langues, puisque deux lexiques disjoints s'y conforment. Mais réalisons une expérience, un néologisme à la Lewis Carroll. En anglais, on peut aisément produire le mot-valise brind, amalgame de br(ain) apocopé et $(m)$ ind aphérésé. Le mot brind, par sa forme même, a la capacité d'activer un réseau notionnel recomposé pertinent : le cerveau en tant que moteur actif de l'esprit, ou l'esprit dans sa dimension cérébralement incarnée. Le mot brind, malgré sa transparence conceptuelle, conserve de son incongruité notionnelle en raison du caractère innovant de son signifiant et de l'absence d'historique dialogique : l'interprétant ne dispose d'aucune notion construite à travers l'expérience de discours antérieurs, et ce mot ne s'extrait de rien ; seule la motivation signifiante en oriente l'interprétation. Avec cette réserve, force est de constater que ce mot fonctionne: par sa forme incarnable, il est capable de faire penser un concept corps-esprit unifié, non préexistant dans le lexique et ne correspondant pas à un objet du monde préalablement identifié. Au contraire, c'est le néologisme même qui suggère qu'antérieurement nous passions peut-être à côté d'une manière pertinente de «voir » ou produire les choses faute de mode d'accès. En cela, le lexique est porteur d'une idéologie conceptuelle : il impose des routines telles que la désintégration brain / mind ou, dans le cas contraire, l'intégration brind; il sédimente au niveau des unités de bases des stéréotypes discursifs tels que la disjonction cerveau / esprit. Ainsi, on s'imagine penser inventivement en recomposant librement un lexique désignatif intellectuellement neutre alors qu'en réalité on recompose des fragments conceptuels formellement extraits de discours antérieurs et enregistrant des éléments de sens issus de ces discours; le lexique est le fonds commun de nos préjugés, et son utilisation, la clé vocale de leur réactivation. Le jeu de mot, qu'il soit d'ordre syntaxique (louer 1 vs louer 2 selon l'insertion discursive) ou formel (brain + mind = brind), a pour vertu première de faire jouer de manière non conventionnelle la dynamique de focalisation attentionnelle et de la mettre en exergue en tant que telle. On retrouve pour une part l'hypothèse Sapir-Whorf selon laquelle une langue, et en particulier son lexique, fournissent une vision conceptualisée du monde construite dans un rapport à un environnement spécifique avec ses conditions matérielles et son mode de vie. Mais on va plus loin en insistant sur l'importance de l'évolution historique du lexique en tant que système de citations dialogique fragmentées du côté du signifié et en tant qu'intervension dans le monde (plutôt que représentation) pour le signifiant.

Dans certains cas, le lexique porte dans sa forme même une ou plusieurs indications procédurales, de nature motrice et sensorielle, sur la manière dont le sens est activé. Dans le lexique anglais, il existe des matrices consonantiques communes à des groupes lexicaux fédérés par un mode d'accès empirique fédérateur (Bottineau 2008). Par exemple, on peut montrer que la séquence $<$ sp $>$ est liée à la notion de rotation, d'éjection par force centrifuge et de projection : elle est commune à spin « rotation rapide », « essorage », span « envergure » 
(ailes déployées d'un oiseau), spear «javelot», spit «cracher», spew «vomir », speak «parler ». L'élément formateur $s p$ - souligne un élément d'expérience sensorimotrice, ici la centrifugation, caractéristique de l'action (spin), du comportement d'un objet (spool «bobine ») ou de sa manipulation par un humain (spoon «cuiller»: s'utilise par un mouvement rotatif ; cf spear «javelot»). Cette convocation est un possible sémiotique fourni comme modèle de conceptualisation fédérateur par la forme du lexique-modèle externe aux sujets : chaque sujet n'est pas tenu de réaliser l'interprétation connotative du submorphème pour que le lexique dénotatif fonctionne (spoon peut être compris globalement), aussi chacun réalise-t-il une appropriation personnelle du mot selon le paramétrage du degré d'analyse par les pratiques verbales rencontrées. En l'occurrence, l'accès au submorphème est favorisé par la connaissance des chansons enfantines (nursery rhymes), qui par le jeu de la métrique et des rimes en sont richement décorées: une pratique soutenue de ces rites vocaux en milieu scolaire et familial et dans le contexte d'un rapport affectif positif en favorise la prise en compte sémiotique, en particulier chez les experts qui par la suite écrivent, enseignent, prennent la parole écrite ou orale publiquement, et contribuent à entretenir les manifestations formelles de cette cohérence, qui continuera à se présenter comme modèle sémiotique externe quand bien même seule une petite minorité de la communauté se l'approprie effectivement.

Cela signifie qu'il ne s'agit pas de motivation phonosymbolique comparable à l'onomatopée comme on l'a longtemps cru. Rien dans la langue actuelle ne prédispose <sp-> à évoquer la centrifugation, et il n'y a aucune raison de considérer que ce groupe consonnes soit la transduction vocale ou auditive d'une boucle motri-sensorielle non verbale, même avec toutes les synesthésies imaginables : en soi, <sp-> semble « arbitraire » au sens saussurien. Il existe toutefois un modèle, la théorie sémio-génétique de Philps (2009 et 2010), selon lequel ces matrices, traçables jusqu'au Proto-Indo-Européen reconstruit, auraient eu initialement la capacité à évoquer le geste moteur dont l'évènement acoustique résulte, puis auraient par la suite évolué par corrélations articulatoires et relations de contiguïté. Par exemple, le marqueur submorphémique $\langle\mathrm{kn}-\rangle$, dans le lexique le plus ancien, se corrèle à l'articulation mandibulaire, comme en breton actuel genou «bouche» ou en anglais to gnaw one's teeth «faire grincer ses dents ». Par la suite, les mots en $k n$ / gn en viennent à désigner d'autres articulation en s'éloignant de celle de départ: (i) articulations corporelles comme le genou (knee, genou en français) ; (ii) articulations formées par le rapport prothétique liant le corps à l'objet: knife «couteau »; (iii) articulations formées par l'objet lui-même : russe kniga « livre ». Si une telle hypothèse se vérifiait, il existerait un fondement neurologique : chez le chimpanzé, le système de contrôle moteur de la préhension manuelle est activé par la vision d'un objet repéré à proximité de la main; et lorsque le singe apprend à manipuler des objets avec un bâton, ce système s'active si l'objet est distant mais qu'un bâton est disponible pour le rapprocher (Frith 2007). Ce que suggère la propagation de $<\mathrm{kn}->$ de proche en proche, c'est le recrutement par la motricité centrale $<\mathrm{kn}->$ (mandibulaire) de motricités analogues proches, puis plus distantes et médiées par des objets : le cerveau s'organise en fonction de l'analogie liant le contrôle du geste articulatoire vocal $<\mathrm{kn}->$ et celui de gestes articulatoires non verbaux analogues impliquant d'autres membres et la manipulation ou le comportement d'objets extérieurs ; il fait ainsi d'une pierre plusieurs coups en centralisant autour d'un nombre limité de programme moteurs le contrôle d'actions hétérogènes mais structurellement analogues et de facto contrôlables par le même système de commande. Cette organisation s'explique également par le processus de construction du cortex lors de son développement.

Tout ceci illustre la manière dont (i) une boucle motri-sensorielle profile un processus cognitif par sa forme même, et (ii) un processus historique d'extension analogique recrute des processus biosémiotiques hétérogènes sous le même profil, créant dans le lexique des effets d'unification par les signifiants inexplicables par le phonosymbolisme classique et non 
imputable à l'expérience subjective individuelle du rapport mot / chose. En anglais, les submorphèmes de ce type sont nombreux: <sp-> pour la centrifugation, <st-> pour l'immobilisation et la rigidité (stay, stop, still...), <sw-> pour l'oscillation pendulaire (swing, swim, sweep...), tw- pour la même oscillation accélérée constitutive de la binarité par le geste (twitch, two, twin, between...), et bien d'autres. En français, ces structures existent, mais l'hybridation des sources romanes et germaniques a considérablement dilué leurs manifestations. Dans les langues sémitiques, il existe un système pratiquement universel de corrélations motri-sensorielles entre l'articulation phonatoire et l'accès phénoménologique à l'expérience des objets et processus, que Bohas 2006 décrit et théorise dans le cadre de la théorie matrices - étymons - racines. Une telle organisation du lexique n'est pas en soi nécessaire, nombre de langues s'en dispensent, mais elle est efficace, elle est de nature à faciliter la corrélation des mécanismes biosémiotiques verbaux et non verbaux, et donc elle peut plaire à une communauté parlante en satisfaisant ses besoins, auquel cas ses traces se sédimentent de manière plus ou moins cohérente et massive dans le lexique - faiblement en français, moyennement en anglais, fortement en arabe. Il faut donc y voir une motivation souple, variable en typologie, mais pas un déterminisme; et il ne faut surtout pas récuser le système dans une langue donnée sous prétexte que l'on ne retrouve pas ailleurs ce que le modèle semble prédire : dans une approche non déterministe des comportements langagiers comme éléments de l'histoire culturelle, les cohérences locales, à la fois aléatoires et motivées, n'ont pas à se reproduire mécaniquement.

Du côté de la «grammaire », on constate également que les morphèmes sont structurés par des combinaisons de «submorphèmes » de nature à profiler le processus de construction du sens abstrait. Prenons l'exemple des démonstratifs anglais this et that «ce». L'anglais articule ici trois oppositions :

(i) $w h$ - «amémoriel » vs th- «mémoriel». wh- «instruit» l'interprétant que l'entité recherchée n'est pas disponible en mémoire de travail (what book?) alors qu'avec th- elle l'est (that book). Ceci donne lieu à diverses paires : lieu inconnu where « où »vs lieu connu there « là »; moment inconnu when « quand » vs moment connu then « alors ». Hors de telles oppositions, l'instruction mémorielle en th- se retrouve dans l'article défini the «le», l'adverbe de manière thus «ainsi » (= de la manière que nous avons évoquée), le concessif though «bien que ».

(ii) $s$ « continu / présent »/ $t$ « discontinu / interrompu / passé ». $s$ instruit l'interprétant de repérer comme présent et continu un élément de sens ciblé, $t$ de le considérer comme révolu. Ceci concerne la conjugaison des verbes (plays / played), l'approbation (présente : yes « oui »; passée, concession : yet «pourtant»), la négation (immédiate : no, reprise : not) et d'autres. Pour les démonstratifs, this book signifie que l'objet repéré (th-) est catégorisé comme livre au moment même où on le dénomme, et que l'interprétant doit en découvrir la nature catégorielle à travers l'occurrence désignée. That book signifie que l'occurrence désignée est prédéfinie et reconnue comme book, la chose étant présentée admise entre les interlocuteurs et non renégociable. De très nombreuses études ont décrit les implications émotionnelles et interactionnelles de cette alternance.

(iii) $i$ «proximal », «immédiat» vs $a$ «distal». Dans le cadre de ces contrastes, $i$ instruit l'interprétant de conjoindre deux entités, et $a$ de les disjoindre. Ce contraste se manifeste dans le système des articles (the / a), celui des auxiliaires (be / have), des interrogatifs (which / what). Dans le cas de this et that, outre les effets matériels de proximité et distance spatiale, se trouve le fait que les processus marqués par les marqueurs th- (reprise mémorielle) et $-s /-t$ posent un problème de synchronisation opératoire : th- et $-s$, tous deux présents, coïncident $(i)$ avec l'instant de parole; en revanche, th- (acte présent de remémoration) et - $t$ (acte passé de dénomination) ne coïncident pas $(a)$ dans l'instant présent où ils sont vocalisés ensemble. 
Au niveau des marqueurs complets, il apparait que this est porteur d'un processus complexe articulant la remémoration d'une entité mémorisée $(t h-)$ et son association immédiate $(i)$ à une catégorisation présente $(-s)$ pouvant être explicitée par le nom qui suit, alors que that articule une remémoration du même type à une catégorie passée $(-t)$ dont l'établissement, présupposé, est disjoint de l'instant de remémoration $(a)$. Pour certains marqueurs, on peut soupçonner un haut degré de solidarité entre le geste articulatoire et la forme schématique du processus interprétatif associé, le « cognème », par exemple la jonction pour $i$, la disjonction pour $a$, la poursuite pour s, l'interruption pour $t$. Dans d'autres cas, cette corrélation est beaucoup moins évidente, comme pour le rapport de th à la mémoire ou de $n$ à la négation. L'idée est que dans ces environnements morphologiques fermés que sont les microsystèmes grammaticaux, où les effets d'alternances et d'analogies sont aisés à détecter en raison du nombre limité de participants, il a existé ou existe encore une solidarité biosémiotique entre le geste signifiant et le produit signifié, encore manifeste pour certain, et moins pour d'autres en raison des évolutions divergentes des gestes articulatoires et des formes schématiques. Il en découle des assemblages d'opérateurs cognémiques dont certains semblent «motivés », d'autres pas ; et globalement, des opérateurs grammaticaux qui affichent en toute transparence dans leur signifiant incarnable leur propre «notice de montage» cognitif, biosémiotique. Ce dispositif permet aux marqueurs de centraliser dans leur matrice biomécanique le contrôle de processus sémantiques abstraits, grammaticaux, correspondant à des opérations de mise en relation et de repérages complexes sans invoquer des objets et évènements dont l'expérience soit directement remémorable à travers les enregistrements lexicaux de fragments dialogiques. L'abstraction du sens à construire et le défaut d'expérience matérielle tangible de «la deixis », «le passé », "l'irréalité », sont corrigés par la matérialité des actes signifiants par lesquels on les produit, ce qui résout le problème par une solution biosémiotique intégrée aux formes en tant que gestes.

La chose semble difficile à admettre dans le contexte d'une idéologie linguistique dominée par l'arbitraire du signe désincarné et l'autonomie des systèmes lexicaux, morphologiques, syntaxiques et sémantiques. Pourtant le phénomène est très général dans la grammaire de l'anglais, il a été largement décrit, on peut l'éprouver en posant des contraintes méthodologiques qui tiennent compte (a) de la variation de la réalisation des voyelles selon la structure syllabique et accentuelle (cf. what et that) et (b) des conditions d'activation de la semiosis submorphémique (le $a$ de hat «ne veut rien dire » parce qu'il n'y a pas de contraste hit / hat en système : la semiosis du phonème n'est pas automatique, elle est activée dans le cadre d'oppositions et de réseaux analogiques).

Les submorphologies lexicale et grammaticale sont complémentaires, elles opèrent souvent conjointement : dans le contraste spin « rotation rapide» / span «envergure », <sp-> instruit le dénominateur commun (la centrifugation) et $i / a$, le contraste opération / résultat (spin saisit la rotation au moment de son exécution, span le produit de son résultat: le déploiement), selon un rapport de variable $i / a$ à constante $<\mathrm{sp}>$. Pour le $-n$, on ne dispose pas de contrastes robustes qui permettent de certifier que sa présence participe à la sémiosis. De manière générale, les marqueurs lexicaux présentent un ou plusieurs marqueurs submorphémiques sans nécessairement se prêter à une décomposition totale. Les langues sémitiques présentent une illustration spectaculaire du croisement en peigne des submophologies lexicale et grammaticale, avec d'un côté les schèmes consonantique pour la classification phénoménologique des expériences sensorimotrices, et de l'autre les radicaux vocaliques pour la classification des mots et la conjugaison des verbes.

\section{La fabrique de la langue}


Parler dans une langue comme le français, le breton ou le basque, c'est mobiliser un ensemble de ressources comportementales cohérentes : le lexique, la morphologie grammaticale, la syntaxe et la prosodie, les orchestrer selon un protocole cohérent routinier et appris, et produire des expériences psycho-sémantiques distribuées à soi-même (parole réflexive endophasique) et à autrui (parole communicative exophasique), les deux n'étant pas néssairement discriminés ni consciemment visés. Parler, c'est (se) faire penser (Bottineau 2010b), et (se) faire être par là-même en distribuant intersubjectivement des activateurs de constructions mentales impliquant des savoirs collectifs et des rôles intersubjectifs et sociaux formatés par l'usage et formalisés par le système incarnable. Par le lexique, on active des notions dialogiques, des catégories profilées et étendues par l'historique des interactions verbales. Par la morphologie grammaticale, on active des processus de combinaison et de repérage du type de ceux illustrés supra par this et that.

Par la syntaxe, on exécute des enchaînements protocolaires des deux classes de processus précédents. Leur organisation distingue les langues : en français, par l'enchaînement groupe nominal / groupe verbal, on progresse d'un thème vers un rhème, à savoir, d'une base de connaissance partagée (par la perception, le discours, le savoir préalable) vers une contribution intersubjectivement distinctive (Le feu est vert - on était deux à savoir qu'il y avait un feu, mais c'est moi qui annonce son passage au vert). Cette progression est un procole obligatoire, qui donne lieu à simulation même en cas de non-pertinence en situation : il pleut (on est deux à savoir qu'il existe un environnement ouvert «il » extérieur au couple interlocutif je/tu, mais c'est moi qui proclame sa manifestation «pleut»). Sa répétition en fait un cycle procédural et une horloge biosémiotique qui structure la continuité des actes de production sémantique : de même que nous sommes entraînés à marcher en enchaînant des transitions cycliques entre états instables, nous sommes entraîner à parler, penser et nous autodéterminer psychologiquement en enchaînant des actes biosémiotiques cycliques structurés par la syntaxe. Les fondements neurologiques et biomécaniques des cycles syntaxiques sont connus, mais pas leurs effets sur la formation et l'inscription psychosociale des sujets selon la langue pratiquée.

La syntaxe du basque est autre : «Je lis le journal » se dit Nik egunkaria irakurtzen dut 'moi, journal-le, lisant le-je' ; la procédure ordinaire consiste à convoquer les arguments (moi, journal) en leur assignant des fonctions relationnelles marquées par des cas (ici l'ergatif $-k$ pour l'intervenant $n i$ «moi » sur l'objet journal, lui-même à l'absolutif non marqué et relié à rien). Puis apparaît le procès qui les confronte ('lisant'), et enfin l'auxiliaire agglutinant final, $d u t$, qui incorpore les diverses marques de personne (ici, double accord $d$ - pour le journal et - $t$ pour moi) et les spécifications de temps (présent vs passé), de modalité (réalité vs hypothèse) et d'autres au gré des choix énonciatifs ${ }^{8}$. Par ce scenario, le sens s'élabore progressivement en produisant des arguments et en les concentrant in fine dans l'auxiliaire: on parcourt l'arborescence des branches analytiques vers le tronc synthétique par convergence. En fin de course, il est même possible d'adresser l'ensemble de la tirade à un allocutaire spécifié, marqué dans l'auxiliaire ou le verbe sous la forme d'un élément formateur supplémentaire na ( $2^{\mathrm{e}}$ personne féminine) ou ka (masculine) : Nik egunkaria irakurtzen dinat 'moi, journal-le,

\footnotetext{
${ }^{8}$ Les éléments formateurs changent de forme et de place au gré des paramétrages : dut (présent) devient nuen au passé, nuke à l'hypothétique, nukeen à l'hypothétique passé. La voyelle - $u$-, marqueur d'un couple absolutif + ergatif, est remplacée par - $i$ - si on ajoute un élément datif intermédiaire : diot «je le lui », où -o-figure le datif « lui »; passé nion, hyp. nioke, niokeen. Le principe est que chaque agglutination est un réseau dont les parties (les éléments formateurs) varient par leur forme et leur position selon l'état général de l'ensemble; cette variation est inégale selon les rangs personnelles $\left(1^{\mathrm{e}}\right.$ et $2^{\mathrm{e}}$ personne vs $\left.3^{\mathrm{e}}\right)$ et les cas (le datif est le plus robuste, l'absolutif le plus fragile). Il ne faut surtout pas voir dans ce système un jeu de lego où chaque partie serait stable par sa position, sa forme et sa couleur.
} 
lisant le-toi-je' «(je te dis à toi, femme, que) je lis le journal ${ }^{9}$. Le rôle de cette conjugaison allocutive est de faire de l'allocutaire spécifié le motif de la prise de parole (le responsable de l'acte de révélation, ou de l'émotion qui le porte) et éventuellement d'afficher en public cette relation spéciale (en cas de présence detémoins). Apprendre le basque, c'est apprendre à dominer la complexité et variation de cette procédure, exactement comme aux échecs on apprend le mouvement des pièces en songeant à l'échec et mat terminal. A défaut de comprendre le «sens » de cette démarche, on passe à côté de la procédure sémiotique qui caractérise cette langue, et on ne sait pas (se) faire advenir à soi-même et à autrui en basque.

La syntaxe du breton est encore autre : à l'opposé du français, elle commence toujours par une focale allocutive, à savoir le constituant sémantique «urgent», par lequel on agit prioritairement et efficacement sur l'interprétant par l'acte de parole. «Je m'appelle Didier» se dit Didier eo ma añv 'Didier est mon nom', "Il fait chaud» se dit Tomm eo an amzer 'Chaud est le temps', et «Je lis le journal» se dit Lenn a ran ar gazetenn 'Lire fais-je le journal » ou Ar gazetenn a lennan 'Le journal lis-je' selon que l'on focalise l'attention interprétative sur l'acte de lecture (implication : je suis occupé) ou la nature de l'objet lu (implication : ce n'est pas le roman que je lis ; ou encore : le journal est momentanément indisponible). Ce protocole exige de la part du locuteur le choix d'un élément initial en fonction d'un effet recherché sur autrui et/ou sur soi-même par l'interaction verbale: la syntaxe bretonne impose mécaniquement une théorie de l'esprit auquel on s'adresse à travers les effets psychologiques et pragmatiques corrélés au choix de telle structure plutôt que telle autre. En matière de co-avènement intersubjectif et pssychosocial, sa mise en œuvre crée des effets de présence à soi-même et à autrui que les bretonnants évoquent régulièrement et qu'ils disent ne pas retrouver en français en raison de la fixité du sujet et du caractère secondaire des variations (par la voix passive et les détachements : moi, je lis le journal). La répétition de ce cycle syntaxique forme les esprits à des habitudes et des manières d'être et d'interagir par le faire verbal: l'horloge protocolaire codétermine de manière distribuée les chemins de la pensée par ceux du discours, mais aussi les chemins de l'être-soi même en rapport au faireautrui. Le développement individuel profilé par ce processus intersubjectif récurrent se moule dans des manières d'être acculturantes qui entrent dans la constitution de l'identité psychosociale des individus et des groupes, et l'intervension extérieure sur la langue bretonne est à l'origine de traumatismes ethnopsychiatriques qui se seraient traduits par l'émergence de pratiques de groupe telles que la «piste».

A travers les trois illustrations qui précèdent, on comprend ce qu'est « une langue »: un système social spécifique de ressources collectives spontanément normées permettant de conduire la production du sens selon un protocole biosémiotique déterminé ; un formalisme interprétable par son incarnation, d'émergence historique spontanée et d'appropriation réflexive et autoritaire postérieure, par des experts. Le recours à la « langue » par la «parole » permet des actes de production sémantique conformes aux normes de l'équipe parlante (profondeur dialogique des notions, complexité des combinaisons et repérages) et de « copenser» soi (endophasie) et/ou autrui (exophasie) en être et en acte, en termes corrélatifs de même profil et selon la même procédure. La parole dans une langue élève ainsi la qualité sémiotique du sens produit par les individus en permettant d'articuler des notions qui enregistrent le savoir collectif du groupe et l'agencent selon des protocoles interactifs complexes, dépassant de loin la «catégorisation » permise par le rapport individuel au monde matériel non médié et symbolisé par le verbe; la parole transfigure continuellement la «pensée » et la «conscience » et fabrique les intersujets, et on ne peut aucunement la réduire à un encodage symbolique de schèmes conceptuels individuels qui lui préexisteraient. La

\footnotetext{
${ }^{9}$ Dut devient dinat parce que le marqueur $n a$ a été inséré dans une position dative disponible : le même énoncé peut exprimer l'attribution «je te lis le journal ». Cette ambiguité est levée en contexte.
} 
parole modifie conjointement le rapport au monde, à autrui et à nous-même ; elle est action coordonnée des corps et orchestration des esprits et du monde qu'ils modifient; en intervenant «dans le monde » sur la relation médiatrice entre sujets relativement aux objets, elle constitue conjointement toutes les instances de manière continue et renouvelée. Chaque « langue » est un mode communautaire, culturellement spécifiant et historiquement fluctuant, de pratiquer cet acte d'autopoïèse.

Pour justifier ceci, il faut resituer la parole et la langue dans le cadre d'une théorie générale de l'expérience. Toute espèce vivante «vit» en entretenant avec l'environnement un rapport profilé : la sélection de signaux par des capteurs sensibles et leur traitement distribué dans le corps, éventuellement centralisé par un cerveau. Cette sélection réduit infiniment la complexité ambiante en ne prélevant qu'un échantillon infime du signal, et sa transduction en signe par les capteurs sensibles et les traitements neuronaux simplifient encore le résultat en inventant des formes, des couleurs (en fonction de seuils critiques activant des réseaux spécifiques), la $3^{\mathrm{e}}$ dimension, en projetant la résistance des matériaux, en incrustant la connaissance des effets du champ gravitationnel sur les déplacements des corps et des objets, etc.; la composition de l'expérience consciente implique autant la déformation du signal entrant que son étoffement par la récupération de données mémorisées de nature motrice, sensorielle multimodale, et symbolique. Le résultat est l'Umwelt de Von Uexküll 1934, il se construit par simplexification selon Berthoz 2009 (= recours à un appareil vivant complexe de simplification du signal transduit), il constitue le domaine consensuel d'interactions caractérisant l'espèce selon Maturana 1978, il produit l'expérience d'un monde au sens théâtral (enaction) - un monde viable où il est possible de connaître et d'agir, et l'évolution de ce couplage oriente l'évolution individualisante du rapport sujet / monde (par les interactions) et espèce / environnement: l'autopoïèse, qui codétermine les sujets et les objets par l'intention relationnelle évolutive et adaptative.

Par rapport à cela, la parole consiste à agir sur ce monde enactif par une dynamique motrice, réelle ou imaginée, en vue d'obtenir par des actes biomécaniques régulés des effets sémiotiques apprenables, intentionnels et reproductibles mais variables et improvisables. Le langage constitue ainsi un sous-domaine conventionnel d'interactions par lequel l'espèce humaine intervient intersubjectivement et collectivement sur son propre couplage environnemental et modifie continûment la relation par laquelle elle cospécifie son monde et elle-même par son action et perception bouclée et mémorisée. Chaque langue est un système de ressources spécifiques, opposable à d'autres, historiquement développé par un groupe humain donné ; sa mise en œuvre oriente dans une direction particulière le devenir de la relation par lequel il co-détermine les évolutions conjointes de lui-même et de son monde par autopoïèse, matériellement et symboliquement : la parole dans la langue infléchit l'histoire du groupe, ses actions, ses idées, sa civilisation, son environnement, dont sa langue elle-même par appropriation réflexive. Chaque individu venant au monde est tenu de s'engager dans le processus global en se formant comme joueur, en apprenant la discipline verbale commune des actes de pensée dans la forme comme dans le contenu, et de se former intellectuellement, psychologiquement, socialement aux pratiques de l'équipe en place de manière à les incorporer pour son propre compte (épiphylogenèse) et les excorporer pour contribuer à la vie du groupe et son évolution. Le rapport du sous-domaine conventionnel verbal au domaine consensuel général est ambigu : le sous-domaine verbal tend à se séparer du domaine général (i) par sa dynamique auto-organisationnelle et systémique (objet de la linguistique distributionnelle et structurale), (ii) par sa capacité à parler du monde et programmer les idées et les actions comme s'il ne faisait pas partie de ce monde et se tenait «à côté » (l'illusion de l'encodagisme représentationnel cognitiviste), (iii) par sa capacité à focaliser les attentions sur les interactions au détriment de l'environnement collatéral et à se substituer à la conscience du 
monde, (iv) par sa capacité à œuvrer comme discipline sémiotique biomécaniquement incarnée de nature à réguler des actes de pensée dans les termes sociaux de l'esprit d'équipe qui naturalise la culture pour tous et pour chacun. Mais ce sous-domaine verbal est lui-même action motrice avec des effets matériels sensibles, et à ce titre la pratique de la parole se fond dans le monde enactif et le modifie en s'y assimilant. Par exemple, la perception visuelle d'une chaise, sans passer par la parole, fait inévitablement appel à la synthèse de tous les savoirs acquis par l'expérience verbale et non verbale: toute connaissance bénéficie de l'expérience des interactions verbales sans nécessairement en convoquer de nouvelle. Ceci fait que la distinction catégorie (psychologique) / notion (lexicale) est fallacieuse et demande à être révisée en termes de continuum.

\section{Conclusion}

Le mot langue, comme tout métaterme, n'échappe pas au fonctionnement du lexique : il opère comme un focalisateur attentionnel et implique l'avènement aux consciences d'un réseau d'associations d'idées constitué dans les pratiques discursives et interactionnelles. Ce colloque même en fait partie, la formulation initiale de son questionnement joue un rôle actif dans la fabrique évolutive du mot et de la notion. Le parole est un mode d'intervension sur le couplage interlocuteurs / environnement dont elle cospécifie les termes relationnels par un enchaînement d'actes biosémiotiques intentionnels. Cette parole est structurée par des pratiques spontanées convergentes formant le savoir-faire spontané, l'épilangue, que l'on peut sélectionner, stabiliser et universaliser par une grammaire prescriptive explicite, un «code de la parole » normée, un métacode. L'impression que le dire encode des conceptualisations du monde résulte des conditions d'exposition propres aux paradigmes de la linguistique structurale d'un côté et de la linguistique cognitive représentationnaliste de l'autre. Cette conception n'est pas «fausse » au sens où on parvient en effet à réaliser des appariements constructions / formalisations conceptuelles du sens dérivées des méthodes de la psychologie. Mais on ne peut s'en tenir à cela. Il importe également de reconsidérer toutes les pratiques verbales comme des interventions humaines coordonnées sur la relation articulant le couplage individu / groupe / espèce / environnements relatifs à ces niveaux. Ces interventions sont des protocoles biosémiotiques socialement convergents et régulés, et ils participent à la coconstitution distribuée et non localisable de tous les termes relationnellement impliqués.

A partir de cette définition, on peut envisager sérieusement la coordination interdisciplinaire de nature à étudier conjointement les manifestations, processus et effets de cette coalition, par les mots, la grammaire, la prosodie, les phrases, sur les sujets, les groupes, les communautés, l'espèce, à tous les niveaux d'historicité et de spatialité, à travers les langues dans leur diversité et le langage dans son unicité universelle. Un tel programme semble de nature à améliorer la compréhension de l'évolution du couplage, de l'auto-détermination de ses orientations, peut-être de sa prise de conscience méta-cognitive et de la prise de contrôle de sa direction pragmatique.

\section{Références}

AUROUX S. \& MAZIERE F. 2006. Hyperlangues et fabriques de la langue, Histoire Epistémologie Langage 28/2.

BAKHTINE M. [1929] 1977. Marxisme et philosophie du langage. Paris : Minuit.

BARBIERI M. 2007. Introduction to Biosemiotics:The New Biological Synthesis. Dortrecht: Springer. 
Bergounioux, G. 2004, Le moyen de parler, Paris : Verdier.

Berthoz A. 2009. La simplexité. Paris: Odile Jacob.

BOHAS G. 2006. The organization of the lexicon in Arabic and other semitic languages. In Perspectives on Arabic Linguistics XVI, Papers from the Sixteenth Annual Symposium on Arabic Linguistics, Cambridge, March, 2002, ed. S. Boudelaa, 1-37. Philadelphia: John Benjamins.

BotTinEAU D. 2006. «La morphosyntaxe allocutive du sens grammatical», Revue de Sémantique et Pragmatique 19/20, juin-décembre 2006, 93-120.

BotTineau D. 2008. "The submorphemic conjecture in English: Towards a distributed model of the cognitive dynamics of submorphemes », Lexis 2, Toulouse.

BotTineAu, D. 2010a, "L'émergence du sens par l'acte de langage, de la syntaxe au submorphème », M. Banniard \& D. Philps (éds), La fabrique du signe, Linguistique de l'émergence, Toulouse : Presses Universitaires du Mirail, Interlangues, linguistique et didactique, 299-325.

BotTineaU, D. 2010b, «Language and enaction », J. Stewart, O. Gapenne, E. Di Paolo (eds), Enaction: toward a new paradigm for cognitive science, MIT, 267-306.

BotTinEAU, D. 2010c, «Les linguistiques cognitives en France, problématiques actuelles », A. O. Tchubaryan, E. I. Pivovar (éds), Les sciences cognitives : questions et perspectives, Actes du $1^{\text {er }}$ séminaire franco-russe en sciences cognitives, Moscou, 21-22 septembre 2010, Académie des Sciences de Russie \& Université d'Etat pour les Sciences Humaines de Moscou, 177-222.

Bottineau, D. 2010d, «Typologie de la déflexivité », D. Bottineau, L. Begioni (éds.), La déflexivité, Langages 2010/2 (178), 89-113.

BotTineau D. \& Roulland D. 2010. «Les constructions impersonnelles en basque », F. Daviet-Taylor, D. Bottineau (dir.), L'impersonnel, Presses Universitaires de Rennes, 243263.

Clark A. 2008. Supersizing the Mind: Embodiment, Action and Cognitive Extension. Oxford: Oxford University Press.

Col G., Aptekman J., Girault S. \& Victorri B. (2010), «Compositionnalité gestaltiste et construction du sens par instructions dynamiques », Cognitextes 5, Numéro spécial AFLiCo 3 Grammaires en Construction(s), consulté le 01/04/2011 : http://cognitextes.revues.org/372

Bernard C., FouRnier J.-M. \& PUECh C. 2010. Histoire des idées sur le langage et les langues, Paris : Klincksieck.

COURSIL J. 2000. La fonction muette du langage. Ibis Rouge Éditions. Guadeloupe: Presses Universitaires Creoles.

COWLEY S. 2007. « Cognitive dynamics and distributed language ». Language Sciences 29(5), 575-583.

COWLEY S. 2009. «Distributed language and dynamics ». Pragmatics and Cognition 17(3), 495-507.

CUlioli A. 1968. «A propos du genre en anglais contemporain ». Les langues modernes, 3, 326-334.

Culioli A. [1981] 1990. «Sur le concept de notion». BULAG $n^{\circ} 8$, repris dans Pour une linguistique de l'énonciation, t.1 : Opérations et représentations, Gap : Ophrys.

DENEZ, P. (1986), «Contraintes formelles de la structuration de la phrase bretonne », in CARRER, Philippe et alii (1986), Permanence de la langue bretonne, de la linguistique à la psychanalyse, Institut Culturel de Bretagne / Skol Uhel ar Vro, Rennes, 19-44.

DOUAY, C. (2000), Eléments pour une théorie de l'interlocution, Un autre regard sur la grammaire anglaise, Presses Universitaires de Rennes. 
DROR I. E. \& HARNAD S. 2006. Cognition Distributed: how cognitive technology extends our minds. Amsterdam \& Philadelphia: Benjamins.

ERARD Y. 1998. "De l'énonciation à l'enaction. L'inscription corporelle de la langue », Cahiers de l'Institut de Linguistique et des Sciences du Langage, $n^{\circ} 11$, Mélanges offerts en hommage à Mortéza Mahmoudian, tome I et II.

Evans V. \& Pourcel S. 2009. New Directions in Cognitive Linguistics. Amsterdam and Philadelphia: John Benjamins.

FAUCONNIER, G. 1984. Espaces mentaux. Aspects de la construction du sens dans les langues naturelles. Paris : Editions de Minuit.

FAUCONNIER G. \& TURNER M. 1998. «Conceptual Integration Networks ». Cognitive Science $22: 133-187$.

FRITH C. 2007. Making up the Mind. Blackwell.

FuCHS C. (dir.) 2004. La linguistique cognitive. Paris: Editions Ophrys.

GALLESE V. AND LAKOFF G. 2005. "The brain's concepts: the role of the sensory-motor system in conceptual knowledge". Cognitive Neuropsychology 22 (3/4): 455-479.

Goody J. 1979. La raison graphique. Paris : Minuit.

Guillaume G. 1964. Langage et science du langage, Paris \& Québec : Nizet \& Presses de l'Université Laval.

HOFFMEYER J. 2008. Biosemiotics : An examination into the Signs of Life and the Life of Signs. Scranton and London: University of Scranton Press.

JANET P. [1889] 2005. L'automatisme psychologique - Essai de psychologie expérimentale sur les formes inférieures de l'activité humaine. Paris : L'Harmattan.

JoUSSE M. 2008. L'anthropologie du geste. Paris : Gallimard, TEL.

KELLER P.-H. 2006. Le dialogue du corps et de l'esprit. Odile Jacob, Paris.

KÖHLER W. 1929. Gestalt Psychology. New York, Liveright. Trad. française: Psychologie de la forme, Gallimard, [1964] 2000.

KRAVCHENKO A. V. 2004. "Essential properties of language from the point of view of autopoiesis". http://cogprints.org/4008/01/PropertiesOfLanguage.pdf.

LADMIRAL J.-R. \& LIPIANSKY E.-M. 1989. La Communication interculturelle. Paris : Armand Colin.

LAKoff G. \& Johnson M. 1999. Philosophy In The Flesh: the Embodied Mind and its Challenge to Western Thought. Basic Books.

LANGACKER R.W. 1987. Foundations of cognitive grammar. Stanford: Stanford University Press.

LAPLANE D. 2000. La pensée d'outre-mots, la pensée sans langage et la relation penséelangage, Institut d'édition sanofi-synthelabo.

LAVIE, R.-J. 2008. «Interspeaker variation and learnability in an exemplar-based productive model », in G. Desgulier, J.-B. Guinard \& J.-R. Lapaire (éds), Du fait grammatical au fait cognitif. From Gram to Mind (2 volumes), Bordeaux: Presses Universitaires de Bordeaux.

LEROI-GOURHAN A. (1964). Le geste et la parole. I. Technique et langage. II. La mémoire et les rythmes. Paris : Albin Michel.

MCGuRK, H., \& MCDONALD, J. (1976). "Hearing lips and seeing voices". Nature, 264, 23-30.

MacNeILAGE P.F. 1998. "The Frame/Content theory of evolution of speech production". Behavioral and Brain Sciences, 21, 499-546.

MCNEILL D. 2005. Gesture and thought. Chicago: University of Chicago Press.

MaturanA, H. (1978), "Biology of language: The epistemology of reality". In G. MiLler and E. LENNEBERG (eds.), Psychology and Biology of Language and Thought: Essays in Honor of Eric Lenneberg, New York: Academic Press, 27-64. 
Maturana H.R. \& VARELA F.J. 1980. Autopoiesis and cognition: the realization of the living. Reidel, Dordrecht.

Merleau-Ponty, M. 1954. Phénoménologie de la perception. Paris : Gallimard.

OGIEN A. 2007. Les formes sociales de la pensée, La sociologie après Wittgenstein. Paris : Colin.

OlivieR G. \& JUAN DE MENDOZA J.L. 2001. "Generation of oculomotor images during tasks requiring visual recognition of polygons". Perceptual and Motor Skills, 92, 1233-1247.

PiAgET J. 1972. Le langage et la pensée chez l'enfant. Neuchâtel: Delachaux et Niestlé.

PHILPS D. 2009. "Conceptual transfer and the emergence of the sign », CogniTextes [En ligne], Volume 2। 2009, mis en ligne le 10 décembre 2009, Consulté le 18 octobre 2010. URL : http://cognitextes.revues.org/180

PHILPS D. 2010. «Stratégies de nomination du corps et émergence du signe linguistique ». In M. BANNIARD \& D. PhiLPS (éds), La fabrique du signe, Linguistique de l'émergence, Toulouse : Presses Universitaires du Mirail, Interlangues, linguistique et didactique, 281298.

RASTIER F. 1993. «La sémantique cognitive : éléments d'histoire et d'épistémologie ». Histoire Epistémologie Langage, 15:1, 153-187.

Rizzolatti G. \& Craighero L. 2004. "The mirror-neuron system". Annual Review of Neuroscience, 27, 169-192.

RuHLEN M. 1994. The Origin of Language: Tracing the Evolution of the Mother Tongue. New York: John Wiley \& Sons.

SADOWSKI P. 2001. "The sound as an echo to the sense: the iconicity of English gl- words". In O. FISCHER \& M. NÄNNY (eds), The motivated sign, iconicity in language and literature 2, Benjamins, 69-88.

SimONDON, G. 1995. L'individu et sa genèse physico-biologique. Paris : Jérôme Millon.

STEFFENSEN S. V. 2009. «Language, Languaging and the Extended Mind Hypothesis. » Pragmatics and Cognition 17(3), 677-697.

STIEGLER B. 1994. La Technique et le temps 1 : La faute d'Epiméthée. Paris : Galilée.

UEXKÜLL J. von [1934] 2004. Mondes animaux et monde humain. Pocket.

VALETTE M. 2006. Linguistiques énonciatives et cognitives françaises. Gustave Guillaume, Bernard Pottier, Maurice Toussaint, Antoine Culioli, collection «Bibliothèque de Grammaire et de Linguistique », Paris : Honoré Champion.

VAlin R. 1981. Perspectives psychomécaniques sur la syntaxe, Laval: Les Presses de l'université Laval, Québec.

VAREla F., Thompson E. \& Rosch E. 1993. The Embodied Mind: Cognitive Science and Human Experience. MIT Press, Cambridge.

VICTORRI, B. 1999. «Le sens grammatical », Langages, 136, 85-105.

VygOTSKY L.S. (1962). Thought and Language. E. KAUFMANN \& G. VAKAR, eds \& trans., Cambridge: MIT Press.

ZIEMKE T. 2003. "What's that thing called embodiment?" In: Proceedings of the 25th Annual Meeting of the Cognitive Science Society. Lawrence Erlbaum. 